\title{
TWO EXTENSIONS OF LUBINSKY'S UNIVERSALITY THEOREM
}

\author{
BARRY SIMON*
}

\begin{abstract}
We extend some remarkable recent results of Lubinsky and Levin-Lubinsky from $[-1,1]$ to allow discrete eigenvalues outside $\sigma_{\text {ess }}$ and to allow $\sigma_{\text {ess }}$ to first be a finite union of closed intervals and then a fairly general compact set in $\mathbb{R}$ (one which is regular for the Dirichlet problem).
\end{abstract}

\section{INTRODUCTION}

This paper primarily discusses orthogonal polynomials on the real line (OPRL) $[36,9,25]$. To set notation, $\mu$ will be a measure of compact support, $\sigma(d \mu)$, on $\mathbb{R}$, positive but not necessarily normalized. Its Lebesgue decomposition is

$$
d \mu(x)=w(x) d x+d \mu_{\mathrm{s}}(x)
$$

where $w \in L^{1}(\mathbb{R}, d x)$ and $\mu_{\mathrm{s}}$ is Lebesgue singular. $\sigma_{\text {ess }}(d \mu)$ will denote $\sigma(d \mu)$ with isolated points removed and $\sigma_{\mathrm{s}}(d \mu)=\sigma\left(d \mu_{\mathrm{s}}\right)$.

$P_{n}(x, d \mu)$ will denote the monic orthogonal polynomials and $p_{n}(x, d \mu)$ the orthonormal polynomials. The Jacobi parameters $\left\{a_{n}, b_{n}\right\}_{n=1}^{\infty}$ are defined by the recursion relation

$$
x p_{n}(x)=a_{n+1} p_{n+1}(x)+b_{n+1} p_{n}(x)+a_{n} p_{n-1}(x)
$$

We note for later use that

$$
p_{0}(x)=\left(\frac{1}{\mu(\mathbb{R})}\right)^{1 / 2}
$$

and that $\left(\|\cdot\|\right.$ means $L^{2}(\mathbb{R}, d \mu)$ norm $)$

$$
\left\|P_{n}\right\|=a_{1} \ldots a_{n} \mu(\mathbb{R})^{1 / 2}
$$

Date: June 11, 2007.

2000 Mathematics Subject Classification. 05E35, 26C10, $26 \mathrm{C} 05$.

Key words and phrases. Lubinsky universality, CD kernel.

* Mathematics 253-37, California Institute of Technology, Pasadena, CA 91125. E-mail: bsimon@caltech.edu. Supported in part by NSF grant DMS-0140592 and U.S.-Israel Binational Science Foundation (BSF) Grant No. 2002068. 
The main focus of this paper is the CD (for Christoffel-Darboux) kernel (for $x, y \in \mathbb{R}$ )

$$
K_{n}(x, y ; d \mu)=\sum_{j=0}^{n} p_{n}(x, d \mu) p_{n}(y, d \mu)
$$

We will often drop $d \mu$ or consider several measures, say $\mu, \mu^{\sharp}$, and use $K_{n}(x, y), K_{n}^{\sharp}(x, y) . K_{n}$ is the integral kernel of the orthogonal projection in $L^{2}(\mathbb{R}, d \mu)$ onto the polynomials of degree at most $n$. So if $Q_{n}(x)$ is such a polynomial, then (the reproducing property)

$$
Q_{n}(x)=\int K_{n}(x, y) Q_{n}(y) d \mu(y)
$$

and, in particular (an expression that $K_{n}$ is the kernel of a projection),

$$
\int K_{n}(x, y) K_{n}(y, z) d \mu(y)=K_{n}(x, z)
$$

Going back to Faber [6], Fekete [7], and Szegö [35], it has been known that there are deep connections between potential theory and asymptotics of polynomials; see Stahl-Totik [34] and Simon [30]. We will be interested especially in the potential theory associated to $E=\sigma_{\text {ess }}(d \mu)$. We will call $E \subset \mathbb{R}$ a regular set if it is compact, regular for the Dirichlet problem on $\mathbb{C}$ and with an equilibrium measure, $d \rho$, of the form $\rho(x) d x$. Thus, with $C(E)$, the capacity of $E$,

$$
\int_{E} \rho(y) d y=1
$$

and

$$
G_{E}(x)=\int_{E} \log |x-y| \rho(y) d y-\log C(E)
$$

is continuous on $\mathbb{C}$ with

$$
G_{E}(y)=0 \text { if } y \in E \quad G_{E}(z)>0 \text { if } z \notin E
$$

Stahl-Totik introduce the important notion of regular measure: If $\mu$ is a measure of compact support, we call it regular if and only if

$$
\lim _{n \rightarrow \infty}\left(a_{1} \ldots a_{n}\right)^{1 / n}=C(E)
$$

where $E=\sigma_{\text {ess }}(d \mu)$. (They use $E=\sigma(d \mu)$, but since $\sigma(d \mu) \backslash \sigma_{\text {ess }}(d \mu)$ is countable, it has zero capacity, and so there is no difference.) One reason this is natural is that it is always true that

$$
\limsup _{n \rightarrow \infty}\left(a_{1} \ldots a_{n}\right)^{1 / n} \leq C(E)
$$


(An elegant way to see this (see Widom [43] or [30]) is to note that when $\mu(\mathbb{R})=1, a_{1} \ldots a_{n}=\left\|P_{n}\right\|_{2} \leq\left\|T_{n}\right\|_{\infty}$ with $T_{n}$ the Chebyshev polynomial for $E$ and use Szegö's theorem [35] that $\lim \left\|T_{n}\right\|^{1 / n}=C(E)$.)

More generally than (1.12), one has the results of Stahl-Totik [34] (see also [30]) that

Theorem 1.1 ([34]). If $E=\sigma_{\mathrm{ess}}(d \mu)$ and $\mu$ is regular, then

$$
\limsup _{n \rightarrow \infty}\left|p_{n}(z, d \mu)\right|^{1 / n} \leq e^{G_{E}(z)}
$$

uniformly on compact subsets of $\mathbb{C}$. In particular, if $E$ is regular, for any $\varepsilon$, there is a $\delta$ and $C$ so

$$
\sup _{\operatorname{dist}(y, E) \leq \delta}\left|p_{n}(y, d \mu)\right| \leq C e^{\varepsilon n}
$$

One connection between $K$ and $\rho$ is (an analog of Theorem 8.2.6 of [25]; see Simon [32]):

Theorem 1.2 ([32]). For any regular measure $\mu$,

$$
\frac{1}{n} K_{n}(x, x) d \mu \rightarrow d \rho_{E}
$$

the equilibrium measure for $E=\sigma_{\mathrm{ess}}(d \mu)$. In (1.14), the convergence is weakly as probability measures on $\operatorname{supp}(d \mu)$.

If $E$ is regular, if $d \mu$ given by (1.1) is regular, and if $\frac{1}{n} K_{n}(x, x)$ has a uniform limit as $n \rightarrow \infty$ for $x \in I$ some open interval, then by (1.14), that limit must be $\rho(x) / w(x)$ (and $w(x)$ must be continuous and nonvanishing on $I)$. This motivates

Definition. We say that $\mu$ has normal limits on a closed interval $I=$ $[a, b]$ if and only if for any $x_{n} \rightarrow x \in I$,

$$
\frac{1}{n} K_{n}\left(x_{n}, x_{n}\right) \rightarrow \frac{\rho_{E}(x)}{w(x)}
$$

with convergence which is uniform in the sense that for any $\varepsilon$, there is $N$ and $\delta$ so that $n \geq N$ and $\left|x_{n}-x\right|<\delta$ implies the difference in (1.15) is less than $\varepsilon$.

Normal limits for $x_{n} \equiv x$ has an ancient history for orthogonal polynomials on the unit circle (OPUC) and for $E=[-1,1]$, going back to Szegő with important contributions by Erdös, Turán, and Freud. This history is discussed in the fundamental paper by Máté-Nevai-Totik [20] who obtained very strong results on pointwise convergence for $\mu$ 's supported on $[-1,1]$ or OPUC supported on $\partial \mathbb{D}$. The refinement of allowing $x_{n} \rightarrow x$ is one critical idea in a recent wonderful paper of 
Lubinsky [17] who provides a result on off-diagonal behavior of $K_{n}$ also:

Theorem 1.3 ([17]). Suppose $I=[a, b]$ is a closed interval in $(-1,1)$ and $d \mu$ is a regular measure with support $[-1,1]$ and that $\operatorname{supp}\left(d \mu_{\mathrm{s}}\right) \cap I=\emptyset$. Suppose on $I, d \mu(x)=w(x) d x$ with $w$ continuous and nonvanishing. Then $\mu$ has normal limits on $I$, and for $x_{0} \in I$ and $\alpha, \beta \in \mathbb{R}$,

$$
\lim _{n \rightarrow \infty} \frac{K_{n}\left(x_{0}+\frac{\alpha}{n}, x_{0}+\frac{\beta}{n}\right)}{K_{n}\left(x_{0}, x_{0}\right)}=\frac{\sin \left(\pi \rho\left(x_{0}\right)(\beta-\alpha)\right)}{\pi \rho\left(x_{0}\right)(\beta-\alpha)}
$$

uniformly if $|\alpha|,|\beta| \leq A, x_{0} \in I$ for any $A>0$.

Remarks. 1. Continuity "on I" here means continuous at each point in $I$ as a function on $[-1,1]$, that is, continuity at $a, b$ involves values of $w$ outside but near $[a, b]$. Thus, the continuity hypothesis is nonvacuous if $a=b$, and the theorem is interesting in that case.

2. The earliest results of the form (1.16) come from the random matrix and Riemann-Hilbert literatures; see [13].

3. Lubinsky [17] does not use $\rho\left(x_{0}\right)=\left(\pi \sqrt{1-x_{0}^{2}}\right)^{-1}$ for (1.16), but scales using $w(x) K_{n}(x, x) \sim n \rho(x)$. This gives a form that makes contact with the Riemann-Hilbert literature and is also suitable for end points and Freud weights.

4. As we will explain in Section 4, Levin-Lubinsky [16] use (1.16) to control asymptotics of zeros of $p_{n}$.

Our goal in this paper is to extend Theorem 1.3 in two ways:

(a) Instead of requiring $\sigma(d \mu)=[-1,1]$, we want to allow $\sigma_{\text {ess }}(d \mu)=$ $[-1,1]$, as is natural if one makes assumptions on $\left\{a_{n}, b_{n}\right\}_{n=1}^{\infty}$ rather than directly on $d \mu$.

(b) We want to replace $[-1,1]$ by a general finite gap set.

A third important extension involves (1.16) pointwise for a.e. $x_{0} \in I$ for situations where $d \mu$ obeys a local Szegó condition on $I$. I had intended to combine Lubinsky's strategy with ideas of Máté-NevaiTotik [20] and especially Totik [37], but I was informed by Totik that Findley [8] and he [39] have results along this line. So I decided to focus here only on (a) and (b).

Both of these extensions, while important, are not especially difficult. Because of Lubinsky's clever inequality (see (4.1)), it is only necessary to find a suitable universal model for $E$ and to control the diagonal kernel. 
A key will be to relate $K_{n}(x, x)$ to the Christoffel function,

$$
\lambda_{n}\left(x_{0}\right)=\min \left\{\|Q\|_{L^{2}(\mathbb{R}, d \mu)}^{2} \mid Q\left(x_{0}\right)=1, \operatorname{deg} Q \leq n\right\}
$$

The minimizer is

$$
Q_{n}\left(x, x_{0}\right)=K\left(x_{0}, x_{0}\right)^{-1} \sum_{j=0}^{n} p_{j}(x) p_{j}\left(x_{0}\right)
$$

for which

$$
\left.\lambda_{n}\left(x_{0}\right)=K_{(} x_{0}, x_{0}\right)^{-1}
$$

To handle extension (a) will be easy: One can eliminate the point masses distant from $E$ by adding explicit zeros to a trial polynomial and control the point masses near $E$ with some exponential decay.

The key to (b) will be to construct a suitable model that is wellbehaved, and following Lubinsky's strategy (he uses Legendre polynomials as his model), it will be easy to extend Theorem 1.3. Our model will be the measure associated to a point in the isospectral torus associated to $E$ where the analysis will depend on results of Sodin-Yuditskii [33], Peherstorfer-Yuditskii [23], and Christiansen-Simon-Zinchenko $[5]$.

The most subtle part of the model will be establishing (1.16) which will follow from Jost asymptotics. Jost asymptotics are the key to proving clock behavior for zeros in $[27,28,15]$. In a sense, using the Levin-Lubinsky strategy, we can regard (1.16) as a kind of infinitesimal Jost asymptotics.

To obtain control of the diagonal CD kernel, all we will need is a single model, $\mu^{\sharp}$, obeying

(i) $\sigma_{\text {ess }}\left(\mu^{\sharp}\right)=E$

(ii) $w^{\sharp}$ is continuous and nonvanishing in $E$.

(iii) For any closed interval $I \subset E^{\text {int }}$, and $\varepsilon>0$,

$$
\sup _{x \in I} e^{-\varepsilon n} K_{n}^{\sharp}(x, x) \rightarrow 0
$$

(iv) For any closed interval $I \subset E^{\text {int }}$,

$$
\limsup _{\varepsilon \downarrow 0}\left[\limsup _{n \rightarrow \infty} \frac{K_{(1+\varepsilon) n}^{\sharp}(x, x)}{K_{n}^{\sharp}(x, x)}\right]=1
$$

It is known $[43,41,34,30]$ that (ii) implies that $\mu^{\sharp}$ is regular. Of course, we will have

$$
\frac{1}{n} K_{n}^{\sharp}(x, x) \rightarrow \frac{\rho(x)}{w^{\sharp}(x)}
$$


from which (1.20) and (1.21) follow. We state the result in this form to allow for future work where the model focuses on a single point in $E^{\text {int }}$ where $w$ vanishes or blows up at some rate.

In Section 3, we will prove:

Theorem 1.4. Suppose $E$ is a regular set and there exists a model, $\mu^{\sharp}$, obeying (i)-(iv). Let $\mu$ be a measure with $\sigma_{\text {ess }}(\mu)=E$, $\mu$ regular, and $w$ continuous and nonvanishing on $I=[a, b] \subset E^{\mathrm{int}}$. Suppose that $\sigma_{\mathrm{s}}(\mu) \cap I=\emptyset$. Then for any $x_{n} \rightarrow x \in I$,

$$
\frac{K_{n}\left(x_{n}, x_{n}\right)}{K_{n}^{\sharp}\left(x_{n}, x_{n}\right)} \rightarrow \frac{w^{\sharp}(x)}{w(x)}
$$

uniformly in the sense discussed after (1.15).

Remark. By (1.22), we have normal behavior on $I$.

In Section 4, we will prove:

Theorem 1.5. Suppose $E$ is a regular set and there exists a model, $\mu^{\sharp}$, obeying (i)-(iv) so that $K^{\sharp}$ obeys (1.16) uniformly for $x$ in compacts of $E^{\text {int }}$, and $|\alpha|,|\beta|<A$. Let $\mu$ be a measure with $\sigma_{\mathrm{ess}}(\mu)=E$, $\mu$ regular, and $w$ continuous and nonvanishing on $I=[a, b] \subset E^{\mathrm{int}}$. Suppose that $\sigma_{\mathrm{s}}(\mu) \cap I=\emptyset$. Then $K$ obeys (1.16) uniformly on $I$ and $|\alpha|<A$, $|\beta|<A$.

Given Theorem 1.4, we will obtain Theorem 1.5 by following Lubinsky's argument virtually word for word. It will also, following LevinLubinsky, imply uniform clock behavior of the zeros in $I$ in the sense of Last-Simon [15] (if $a<b$ ).

In Section 2, we will obtain $\mu^{\sharp}$ obeying (i)-(iv) when $E$ is a finite union of intervals and (1.16), therefore accomplishing extensions (a) and (b).

All our arguments extend with little change to finite gap OPUC and to zeros of paraorthogonal polynomials [3, 4, 10, 12, 29, 44].

During the preparation of this manuscript, I learned that Totik [39] was also working on extending Lubinsky universality to general sets. After I finished the above and Sections 2-4 below, Totik and I exchanged manuscripts. His technical methods are different from what I do in Section 2. After I got his manuscript, I realized that Lubinsky's inequality ((4.1) below) is so strong that it is easy to go from finite gap to general compact sets and prove

Theorem 1.6. Let $E \subset \mathbb{R}$ be an arbitrary regular compact set so that $I=[a, b] \subset E^{\text {int }}$. Let $\mu$ be a measure with $\sigma_{\mathrm{ess}}(\mu)=E$, $\mu$ regular in the sense of Stahl-Totik [34], and $\mu\lceil[a-\varepsilon, b+\varepsilon]$ is purely absolutely 
continuous with $w=\frac{d \mu}{d x}$ continuous and nonvanishing on $[a, b]$. Let $\rho_{E}(x) d x$ be the density for the equilibrium measure for $E$ restricted to $I$ (it is not hard to see $\rho_{E}$ is purely a.c. on I; see [30]). Then uniformly for $x \in[a, b]$,

$$
K_{n}(x, x) \rightarrow \frac{\rho_{E}(x)}{w(x)}
$$

and uniformly for $x_{0} \in[a, b],|\alpha|,|\beta| \leq A$, one has (1.16), and so (as in Section 4 following [16]), clock behavior for the zeros.

Remark. (1.24) is not new. It is essentially in Totik [37].

The proof of this theorem is sketched in Section 5. We will also note there that it suffices to prove the results in Section 2 when each interval has rational harmonic measure so that one can use Floquet theory in place of the more subtle analysis of $[33,23,5]$.

It is a pleasure to thank D. Lubinsky, P. Nevai, and V. Totik for useful correspondence.

\section{MODELS}

Let $E$ be a finite gap set, that is,

$$
E=\bigcup_{j=1}^{k+1}\left[\alpha_{j}, \beta_{j}\right]
$$

where $\alpha_{1}<\beta_{1}<\alpha_{2}<\cdots<\beta_{k+1}$ are reals. Associated with any such $E$ is an isospectral torus of Jacobi matrices defined by the fact that their $m$-functions are Herglotz functions extendable to minimal degree meromorphic functions on the two-sheeted Riemann surface associated to $\left[\prod_{j=1}^{k+1}\left(z-\alpha_{j}\right)\left(z-\beta_{j}\right)\right]^{1 / 2}$. For OPUC, this is discussed, for example, in [26], and for OPRL in [31].

The spectral measure $\mu^{\sharp}$ for any such Jacobi matrix has the form (see $[33,23,5])$

$$
d \mu^{\sharp}=w^{\sharp}(x) d x+d \mu_{\mathrm{s}}
$$

where $d \mu_{\mathrm{s}}$ is a pure point measure with at most one pure point in each gap of $E$ and none in $E$ and

$$
w^{\sharp}>0
$$

and real analytic on $E^{\text {int }}$.

Our goal in this section is the prove the following

Theorem 2.1. Let $\mu^{\sharp}$ be the probability measure associated to a Jacobi matrix in the isospectral torus for $E$. Then, uniformly for $x$ in any interval $[a, b]$ in $E^{\text {int }}$, we have 
(i)

$$
K_{n}^{\sharp}(x, x)=\frac{\rho_{E}(x)}{w(x)} n+O(1)
$$

(ii) Uniformly in $|\alpha|,|\beta|<L$,

$$
\frac{w(x)}{n} K_{n}^{\sharp}\left(x+\frac{\alpha}{n}, x+\frac{\beta}{n}\right)=\frac{\sin \left(\pi \rho_{E}(x)(\beta-\alpha)\right)}{\pi(\beta-\alpha)}+O\left(\frac{1}{n}\right)
$$

We obtain this from results $[33,23,5]$ on Jost solutions, that is, solutions of

$$
a_{n+1} u_{n+2}+\left(b_{n+1}-x\right) u_{n+1}+a_{n} u_{n}=0
$$

an equation solved by

$$
u_{n}=p_{n-1}(x)
$$

Jost solutions, $u_{n}(x)$, solve (2.6) for $x \in E$ and obey

(i)

$$
u_{n}(x)=e^{i n \theta(x)} f_{n}(x)
$$

(ii) On any $I \subset E^{\text {int }}, f_{n}$ is analytic in $x$ and its derivatives are uniformly bounded in $n$ and $x \in I$.

$$
\theta^{\prime}(x)=\pi \rho_{E}(x)
$$

(iv)

(

$$
u_{0}(x)=1
$$

$$
\overline{u_{1}(x)} \neq u_{1}(x)
$$

(vi) The Wronskian

$$
a_{n}\left(u_{n+1} \bar{u}_{n}-\bar{u}_{n+1} u_{n}\right)
$$

is $n$-independent (but $x$-dependent).

(vii) While we do not need it, we note that $f_{n}(x)$ is almost periodic in $n$.

Because of $(2.9) /(2.10), u_{n}$ and $\bar{u}_{n}$ are linearly independent, so $p_{n}$ is a linear combination of $u_{n+1}$ and $\bar{u}_{n+1}$, and thus,

$$
p_{n}(x)=\frac{u_{n+1}-\overline{u_{n+1}(x)}}{u_{1}(x)-\bar{u}_{1}(x)}
$$

Define

$$
g_{n}(x)=\frac{e^{i \theta(x)} f_{n+1}(x)}{\left[u_{1}(x)-\bar{u}_{1}(x)\right]}
$$

so (2.12) becomes

$$
p_{n}(x)=g_{n}(x) e^{i n \theta(x)}+\overline{g_{n}(x)} e^{-i n \theta(x)}
$$


and constancy of the Wronskian becomes

$$
a_{n+1}\left[g_{n+1}(x) \bar{g}_{n} e^{i \theta(x)}-g_{n}(x) \overline{g_{n+1}(x)} e^{-i \theta(x)}\right]=c(x)
$$

$x$-dependent but not $n$-dependent.

We will also need the CD formula

$$
K_{n}^{\sharp}(x, y)=a_{n+1}\left[\frac{p_{n+1}(x) p_{n}(y)-p_{n}(x) p_{n+1}(y)}{x-y}\right]
$$

for $x \neq y$ and its limit at $x=y$,

$$
K_{n}^{\sharp}(x, x)=a_{n+1}\left[p_{n+1}^{\prime}(x) p_{n}(x)-p_{n+1}(x) p_{n}^{\prime}(x)\right]
$$

Proof of Theorem 2.1. By (ii), (iii), and (v), and

$$
p_{n}^{\prime}(x)=i n \theta^{\prime}(x)\left[g_{n}(x) e^{i n \theta(x)}-\overline{g_{n}(x)} e^{-i n \theta(x)}\right]+O(1)
$$

where $O(1)$ is bounded uniformly in $x \in I$ and in $n$. Thus, by (2.14), (2.15), (2.17), and (2.18),

$$
K_{n}^{\sharp}(x, x)=2 i n \theta^{\prime}(x) c(x)+O(1)
$$

Therefore, $\frac{1}{n} K_{n}(x, x)$ converges uniformly on $I$ to $2 i \theta^{\prime}(x) c(x)$, so by (1.14),

$$
2 i \theta^{\prime}(x) c(x)=\frac{\rho_{E}(x)}{w(x)}
$$

and (2.19) is (2.4).

Similarly, by (2.14), (2.8), and (ii),

$$
p_{n}\left(x+\frac{\alpha}{n}\right)=g_{n}(x) e^{i n \theta(x)} e^{i \alpha \pi \rho(x)}+\overline{g_{n}(x)} e^{-i n \theta(x)} e^{-i \alpha \pi \rho(x)}+O\left(\frac{1}{n}\right)
$$

uniformly in $|\alpha|<A$.

Plugging this into (2.16) shows

$$
K_{n}\left(x+\frac{\alpha}{n}, x+\frac{\beta}{n}\right)=2 i n \frac{\sin ((\beta-\alpha) \pi \rho(x))}{\beta-\alpha} c(x)+O(1)
$$

By (2.20) and (2.8), $c(x)=1 / 2 \pi i w(x)$, so (2.22) is (2.5).

\section{Asymptotics of the Diagonal CD Kernel}

Our goal here is to prove Theorem 1.4. The key is an idea of Nevai [21] that lets one exponentially localize CD minimizers, augmented by the regularity ideas of Máté-Nevai-Totik [20], and a simple extension that accommodates discrete spectrum. 
Lemma 3.1. Let $E$ be a regular subset of $\mathbb{R}$. Let $\mu, \mu^{\sharp}$ be two measures both regular for $E$. Let $I$ be a closed interval in $E^{\text {int }}$ with $I \cap\left[\sigma_{\mathrm{s}}(\mu) \cup \sigma_{\mathrm{s}}\left(\mu^{\sharp}\right)\right]=\emptyset$. Fix $C_{1}$ larger than the diameter of $E$. Then for all sufficiently small $\delta$ and each $\varepsilon>0$, there is a constant $C_{2}$ and positive integer $J$ depending on $\mu, \mu^{\sharp}$, and $\varepsilon$ so that for all $m$ and $\ell$,

$$
\lambda_{n}\left(x_{0}, \mu^{\sharp}\right) \leq \sup _{\left|y-x_{0}\right| \leq \delta}\left(\frac{w^{\sharp}(y)}{w(y)}\right) \lambda_{m}\left(x_{0}, \mu\right)+C_{2} e^{\varepsilon m}\left(1-\frac{\delta^{2}}{C_{1}^{2}}\right)^{2 \ell}
$$

for all $x_{0} \in I$ where $n=m+2 \ell+2 J$.

Remarks. 1. While we apply this to $w$ positive and continuous near $x_{0}$, it is stated in a way that should be applicable to situations where $w$ and $w^{\sharp}$ vanish or blow up in the same way (the sup in (3.1) is then interpreted as an essential sup).

2. One should be able, as in Lubinsky [18], to accommodate end points with these methods.

Proof. Let $Q_{m}\left(x, x_{0} ; \mu\right)$ be the optimal trial function for the CD problem at $x_{0}$, that is,

$$
Q_{m}\left(x, x_{0} ; \mu\right)=K_{m}\left(x_{0}, x_{0}\right)^{-1} \sum_{n=0}^{m} p_{m}\left(x, \mu_{0}\right) p_{m}\left(x_{0}, \mu_{0}\right)
$$

By (1.13), there is a $\delta_{1}$ and $C_{3}$ so that

$$
\sup _{\substack{\operatorname{dist}(y, E) \leq \delta_{1} \\ x_{0} \in I}}\left|Q_{m}\left(x, x_{0} ; \mu\right)\right| \leq C_{3} e^{\varepsilon m / 2}
$$

We use here the fact that by $(1.13)$ for $\operatorname{dist}(y, E) \leq \delta_{1}$ with $\delta_{1}$ suitable,

$$
\left|p_{m}(y)\right| \leq c e^{m / 6}
$$

and $m \leq 6 e^{m / 6}$. By shrinking $\delta_{1}$, we can also be sure that

$$
x_{0} \in I, \operatorname{dist}(y, E) \leq \delta_{1} \Rightarrow\left|x_{0}-y\right| \leq C_{1}
$$

We now define $J$ to be the number of points, $x$, in $\operatorname{supp}\left(\mu^{\sharp}\right)$ with $\operatorname{dist}(x, E) \geq \delta_{1}$, which is finite since $\sigma_{\text {ess }}\left(d \mu^{\sharp}\right)=E$, and let $\left\{x_{j}\right\}_{j=1}^{J}$ be those points. We take for our trial polynomials for $\lambda_{n}\left(x_{0}, \mu^{\sharp}\right)$ with $n=m+2 \ell+2 J$,

$$
Q(x)=Q_{m}\left(x, x_{0} ; \mu\right)\left(1-\frac{\left(x-x_{0}\right)^{2}}{C_{1}^{2}}\right)^{\ell} \prod_{j=1}^{J}\left(1-\left[\frac{\left(x-x_{0}\right)}{\left(x_{j}-x_{0}\right)}\right]^{2}\right)
$$


Pick $\delta$ so small that $\{x \mid \operatorname{dist}(x, I) \leq \delta\}$ is disjoint from $\sigma_{\mathrm{s}}(\mu) \cup \sigma_{\mathrm{s}}\left(\mu_{0}\right)$ and $\delta<\delta_{1}$. If $y \in \operatorname{supp}\left(d \mu^{\sharp}\right)$ and $\left|y-x_{0}\right| \geq \delta$, we use (3.3) to see

$$
|Q(y)| \leq C_{4} e^{\varepsilon m / 2}\left(1-\frac{\delta^{2}}{C_{1}^{2}}\right)^{\ell}
$$

where

$$
C_{4}=C_{3} \max _{\substack{\text { such } y \\ x_{0} \in I}}\left|\prod_{j=1}^{J}\left(1-\left[\frac{\left(y-x_{0}\right)}{\left(x_{j} x_{0}\right)}\right]^{2}\right)\right|
$$

(3.3) does not hold at $x_{j}$ but the product $\prod_{j=1}^{J}$ vanishes at such points. Thus, $\int_{\left|y-x_{0}\right| \geq \delta}|Q(y)|^{2} d \mu^{\sharp}(y)$ is bounded by the second term in (3.1).

On the other hand, since the second two factors in (3.4) are bounded by 1 on $\left[x_{0}-\delta, x_{0}+\delta\right],|Q(y)| \leq\left|Q_{m}(y)\right|$, so, since there is no singular spectrum there,

$$
\begin{aligned}
\int_{\left|y-x_{0}\right| \leq \delta}|Q(y)|^{2} d \mu^{\sharp} & \leq \sup _{|x-y| \leq \delta}\left[\frac{w^{\sharp}(y)}{w(y)}\right] \int_{\left|y-x_{0}\right| \leq \delta}\left|Q_{m}(y)\right|^{2} d \mu \\
& \leq \sup _{|x-y| \leq \delta}\left[\frac{w^{\sharp}(y)}{w(y)}\right] \lambda_{m}\left(x_{0}, \mu\right)
\end{aligned}
$$

since adding the part of the integral with $\left|y-x_{0}\right|>\delta$ only makes the integral larger.

Proof of Theorem 1.4. Let $\mu^{*}=\mu^{\sharp}$ be the model obeying (i)-(iv). Once $\delta$ is fixed, we can pick $\eta$ so $\left(1-\frac{\delta^{2}}{C_{1}^{2}}\right)^{2 \eta} \leq e^{-2}$. Then in (3.1), we pick $\ell=\eta \varepsilon m$. The second term in (3.1) is thus $O\left(e^{-\varepsilon m}\right)$.

Divide by $\lambda_{m}\left(x, \mu^{\sharp}\right)$. By (1.20), the second term in (3.1) goes to zero, and so (3.1) implies

$$
\liminf \frac{\lambda_{m}\left(x_{m}, \mu\right)}{\lambda_{m}\left(x_{m}, \mu^{*}\right)} \geq \sup _{\left|x_{0}-y\right| \leq 2 \delta}\left(\frac{w^{*}(y)}{w(y)}\right) \liminf \left(\frac{\lambda_{n}^{*}}{\lambda_{m}^{*}}\right)
$$

Here we used $x_{m}-x_{0} \rightarrow 0$ so $\left|x_{0}-y\right| \leq 2 \delta$ for $m$ large.

Taking $\varepsilon \downarrow 0$, by (1.21), the liminf on the right goes to 1 . Thus, by continuity, taking $\delta \downarrow 0$, we have

$$
\liminf \frac{\lambda_{m}\left(x_{m}, \mu\right)}{\lambda_{m}\left(x_{m}, \mu^{*}\right)} \geq \frac{w^{*}\left(x_{0}\right)}{w\left(x_{0}\right)}
$$

Now we interchange $\mu$ and $\mu^{*}$ in (3.1) but now divide by $\lambda_{n}\left(x_{n}, \mu^{*}\right)$ and use the same arguments to get

$$
\limsup \frac{\lambda_{n}\left(x_{n}, \mu\right)}{\lambda_{n}\left(x_{n}, \mu^{*}\right)} \leq \frac{w^{*}\left(x_{0}\right)}{w\left(x_{0}\right)}
$$


(3.8) and (3.9) complete the proof. All the arguments are uniform in $x_{0} \in I$.

\section{Off-Diagonal CD Asymptotics and Clock Behavior}

In this section, we will prove Theorem 1.5 and note its consequences for zeros of the OPRL. Given Theorem 1.4, this is essentially a straightforward translation of [17] and [16]. We note that earlier Freud [9] had noted that the universality result, (1.16), (which he only had under very restrictive assumptions) implies clock behavior of zeros.

Proof of Theorem 1.5. Let $\mu \leq \mu^{*}$. Then, as noted by Lubinsky [17], for any $x, y$,

$$
\left|K_{n}(x, y)-K_{n}^{*}(x, y)\right|^{2} \leq K_{n}^{*}(y, y)\left[K_{n}(x, x)-K_{n}^{*}(x, x)\right]
$$

This critical result - which we dub Lubinsky's inequality - is proven in a few lines in [17].

Given $\mu$ and $x_{0}$, let $\widetilde{\mu}$ be that multiple of the model $\mu^{\sharp}$ with $\widetilde{w}\left(x_{0}\right)=$ $w\left(x_{0}\right)$. Let $\mu^{\sharp}=\sup (\mu, \widetilde{\mu})$. By the lemma below, $\mu^{\sharp}$ is regular. Thus, by Theorem 1.4, we see that

$$
\frac{K_{n}\left(x_{0}+\frac{\alpha}{n}, x_{0}+\frac{\alpha}{n}\right)}{K_{n}^{*}\left(x_{0}+\frac{\beta}{n}, x_{0}+\frac{\beta}{n}\right)} \rightarrow 1
$$

and

$$
\frac{\widetilde{K}_{n}\left(x_{0}+\frac{\alpha}{n}, x_{0}+\frac{\alpha}{n}\right)}{K_{n}^{*}\left(x_{0}+\frac{\beta}{n}, x_{0}+\frac{\beta}{n}\right)} \rightarrow 1
$$

uniformly if $|\alpha|,|\beta|<A$ and $x_{0} \in I$.

From this and $(4.1)$, we find (dividing by $\left.K_{n}^{*}(y, y)\right)$ that

$$
\frac{K_{n}\left(x_{0}+\frac{\alpha}{n}, x_{0}+\frac{\beta}{n}\right)}{\widetilde{K}_{n}\left(x_{0}+\frac{\alpha}{n}, x_{0}+\frac{\beta}{n}\right)} \rightarrow 1
$$

also uniformly in $|\alpha|,|\beta|<A, x_{0} \in I$. (1.16) for $\widetilde{K}_{n}$ and (4.2)-(4.4) imply (1.16) for $K_{n}$.

Lemma 4.1. Suppose $\mu, \mu^{*}$ are two measures with $\sigma_{\mathrm{ess}}(\mu)=\sigma_{\mathrm{ess}}\left(\mu^{*}\right) \equiv$ $E$ and $\mu \leq \mu^{*}$. Then $\mu$ regular implies $\mu^{*}$ is regular.

Proof. Regularity means

$$
\lim _{n \rightarrow \infty}\left\|P_{n}\left(\cdot, d \mu^{*}\right)\right\|^{1 / n}=C(E)
$$

By (1.12),

$$
\lim \sup \left\|P_{n}\left(\cdot, d \mu^{*}\right)\right\|^{1 / n} \leq C(E)
$$


By $\mu \leq \mu^{*}$ and $\left\|P_{n}(\cdot, d \nu)\right\|=\min \left\{\left\|Q_{n}\right\|_{L^{2}(d \nu)} \mid \operatorname{deg} Q_{n}=n, Q_{n}(x)=\right.$ $x^{n}+$ lower order $\}$,

$$
\left\|P_{n}(\cdot, d \mu)\right\| \leq\left\|P_{n}\left(\cdot, d \mu^{*}\right)\right\|
$$

(4.5), (4.7), and

$$
\lim _{n \rightarrow \infty}\left\|P_{n}(\cdot, d \mu)\right\|^{1 / n}=C(E)
$$

imply (4.5).

Last-Simon [15] define clock behavior and uniform clock behavior. Theorem 1.5 implies

Theorem 4.2. Let $\mu$ be a measure obeying the hypothesis of Theorem 1.5 (so, in particular, E must have a suitable model). If $a=b$, there is clock behavior for the zeros of $p_{n}(x, d \mu)$ at $a$. If $a<b$, there is uniform clock behavior on I. The density of zeros in the clock behavior is $\rho_{E}(x)$.

Remark. In particular, E can be a finite gap set by Theorem 2.1. Thus, we recover and vastly generalize the results of [28].

Proof. We need only follow the ideas of [16]:

Step 1. By the CD formula, $\frac{p_{n+1}(y)}{p_{n}(y)}=\frac{p_{n+1}(x)}{p_{n}(x)}$ for $y \neq x$ if and only if $K_{n}(x, y)=0$. Thus, with $\gamma \equiv \frac{p_{n+1}\left(x_{0}\right)}{p_{n}\left(x_{0}\right)}$, we see by (1.16) that there is a zero of $p_{n+1}(y)-\gamma p_{n}(y)$ within $\frac{1}{n \rho\left(x_{0}\right)}+o\left(\frac{1}{n}\right)$ of $x_{0}$. Since zeros of $p_{n+1}-\gamma p_{n}$ and of $p_{n}$ interlace, we conclude there are zeros of $p_{n}(x)$ within $\frac{1}{n \rho\left(x_{0}\right)}+o\left(\frac{1}{n}\right)$ of $x_{0} \in I$.

Step 2. By the CD formula, for $x \neq y$, if $p_{n}(x)=0$, then $p_{n}(y)=0$ if and only if $K_{n}(x, y)=0$. Thus, by (1.16), there are no two zeros of $p_{n}(x)$ within $(1-\varepsilon) \frac{1}{n \rho\left(x_{0}\right)}$ for any $\varepsilon>0$, that is, we have an $O\left(\frac{1}{n}\right)$ lower bound.

Step 3. By the CD formula and (1.16), if $p_{n}\left(x_{0}+\frac{\alpha}{n}\right)=0$, there exist zeros which are at $x_{0}+\frac{\alpha}{n}+\frac{k}{n \rho\left(x_{0}\right)}+o\left(\frac{1}{n}\right)$ for $|k| \leq K$, and, by Step 2, they are unique.

All these arguments are uniform in $x_{0}$, so we have uniform clock behavior.

\section{General Sets}

As explained in the introduction, this section was written after I saw [39] and realized my results plus Lubinsky's inequality easily allowed one to obtain universality for intervals with continuous a.c. weight in arbitrary compact sets, and also allowed an alternative to Section 2 that only requires Floquet theory. 
Proof of Theorem 1.6. $\mathbb{R} \backslash E$ as an open set is a union of countably many maximal open intervals whose total size, after the two semiinfinite ones are removed, is finite. Thus, for any $q>0$, only finitely many are of size larger than $\frac{2}{q}$, so for any positive integer $q, E_{q}=\{x \mid$ $\left.\operatorname{dist}(x, E) \leq \frac{1}{q}\right\}$ is a finite gap compact set.

Let $\rho(x)$ be the equilibrium density for $E$ (restricted to $I$ ) and $\rho_{q}(x)$ for $E_{q}$. By balayage ideas, $d \rho_{q} \uparrow I$ is nonincreasing, and by some potential theory using the real analyticity of $\rho_{q}$ and $\rho$ on $I$,

$$
\rho_{q}(x) \uparrow \rho(x)
$$

uniformly on $I$ (see $[2,11,14,24,30,34,40]$ for background on the needed potential theory).

For each $q$, pick a multiple, $c_{q} p_{q}(x)$, of the equilibrium measure so $\sup _{I} c_{q} p_{q}<\inf _{I} w(x)$, and let $\mu_{q}=u \vee c_{q} p_{q}$, the measure theoretic max. This is regular for $E_{q}$ by Lemma 4.1. Thus, if $x_{m} \rightarrow x_{0} \in I$,

$$
K_{m}^{(q)}\left(x_{m}, x_{m}\right) \rightarrow \frac{\rho_{q}(x)}{w(x)}
$$

where $w=\frac{d \mu}{d x}$. The trial functions for $\mu_{q}$ used in proving (3.8) can be used for $\mu$, that is, we can get upper bounds directly and see that

$$
\lim \sup n \lambda_{n}\left(x_{n}, \mu\right) \leq \frac{w(x)}{\rho_{q}(x)}
$$

for each $q$. Since (5.1) is uniform on $I$, we see

$$
\lim \sup n \lambda_{n}\left(x_{n}, \mu\right) \leq \frac{w(x)}{\rho(x)}
$$

uniformly for $x \in I$ and $x_{n} \rightarrow x$ (as in Section 3, this means that for any $\varepsilon$, there are $N$ and $\delta$ so if $n \geq N$ and $\left|x_{n}-x\right| \leq \delta$, then $\left.n \lambda_{n}\left(x_{n}, \mu\right) \leq \frac{w(x)}{\rho(x)+\varepsilon}+\varepsilon\right)$.

We can use the polynomials $p_{n}(x, \mu)$ as trial functions for $\mu_{q}$ and so still get (3.1), but unlike in Section 3, we cannot take $\varepsilon$ to zero for $q$ fixed and so not take $\frac{\ell}{n} \rightarrow 0$. Instead for a fixed $q$, there is $\eta(q)$ and we have to take $\ell \geq n \eta(q)$. But as $q \rightarrow \infty, \eta(q) \rightarrow 0$. We obtain

$$
(1+\eta(q)) \liminf n \lambda_{n}\left(x_{n}, \mu\right) \geq \frac{w(x)}{\rho_{q}(x)}
$$

Since $\eta(q) \rightarrow 0$ and $\rho_{q}(x) \rightarrow \rho(x)$, we therefore prove

$$
\lim n \lambda_{n}\left(x_{n}, \mu\right)=\frac{w(x)}{\rho(x)}
$$


The limit argument is essentially one used several years ago by Totik [37], the only difference being that we make uniform assumptions (i.e., continuity) on $w$ and conclude uniformity in (5.5) with variable points.

Now use (4.1) with $K^{*}=K^{q}$, the CD kernel, for $\mu_{q} \geq \mu$ by construction. Replace $(x, y)$ by $\left(x_{0}+\frac{\alpha}{n}, x_{0}+\frac{\beta}{n}\right)$. Divide by $\frac{n \rho\left(x_{0}\right)}{w\left(x_{0}\right)}$ and take $n \rightarrow \infty$ using (1.15) and (1.16) for $\mu_{q}$, and (5.4) to get

$\limsup _{n \rightarrow \infty}\left|\frac{K_{n}\left(x_{0}+\frac{\alpha}{n}, x_{0}+\frac{\beta}{n}\right)}{K_{n}\left(x_{0}, x_{0}\right)}-\frac{\sin \prod \rho_{q}\left(x_{0}\right)(\beta-\alpha)}{\prod \rho\left(x_{0}\right)(\beta-\alpha)}\right|^{2} \leq \frac{\rho_{q}\left(x_{0}\right)}{\rho\left(x_{0}\right)}\left[1-\frac{\rho_{q}\left(x_{0}\right)}{\rho\left(x_{0}\right)}\right]$

Note the mixed $\frac{\rho_{q}}{\rho}$ in the ratio on the left side. Taking $q \rightarrow \infty$ yields the desired limit result.

As a final remark, we note that we can use the same approximation idea to go from finite gap E's with all rational harmonic measures to general finite gap E's. For it is a result of Bogatyrëv [1], Peherstorfer [22], and Totik [38] that any finite gap $E$ with $\ell$ gaps can be approximated by rational harmonic measure sets $E_{q} \supset E$ so $\left|E_{q} \backslash E\right| \rightarrow 0$. The arguments above can get results for general $E$ from the $E_{q}$. The point of this remark is that the construction in Section 2 relies on Jost solutions. For E's with rational harmonic measures, the Jacobi parameters are periodic, and Jost solutions can be constructed with Floquet theory rather than the more elaborate methods of $[33,23,5]$.

\section{REFERENCES}

[1] A. B. Bogatyrëv, On the efficient computation of Chebyshev polynomials for several intervals, Sb. Math. 190 (1999), 1571-1605; Russian original in Mat. Sb. 190 (1999), no. 11, 15-50.

[2] P. Borwein and T. Erdélyi, Polynomials and Polynomial Inequalities, Graduate Texts in Mathematics, 161, Springer-Verlag, New York, 1995.

[3] M. J. Cantero, L. Moral, and L. Velázquez, Measures and para-orthogonal polynomials on the unit circle, East J. Approx. 8 (2002), 447-464.

[4] M. J. Cantero, L. Moral, and L. Velázquez, Measures on the unit circle and unitary truncations of unitary operators, J. Approx. Theory 139 (2006), 430468.

[5] J. Christiansen, B. Simon, and M. Zinchenko, in preparation.

[6] G. Faber, Über Tschebyscheffsche Polynome, J. Reine Angew. Math. 150 (1919), 79-106.

[7] M. Fekete, Über die Verteilung der Wurzeln bei gewissen algebraischen Gleichungen mit ganzzahligen Koeffizienten, Math. Z. 17 (1923), 228-249.

[8] M. Findley, Universality and zero spacing under local Szegö condition, in preparation.

[9] G. Freud, Orthogonal Polynomials, Pergamon Press, Oxford-New York, 1971. 
[10] L. Golinskii, Quadrature formula and zeros of para-orthogonal polynomials on the unit circle, Acta Math. Hungar. 96 (2002), 169-186.

[11] L. L. Helms, Introduction to Potential Theory, Pure and Applied Mathematics, Vol. 22 Wiley-Interscience, New York, 1969.

[12] W. B. Jones, O. Njåstad, and W. J. Thron, Moment theory, orthogonal polynomials, quadrature, and continued fractions associated with the unit circle, Bull. London Math. Soc. 21 (1989), 113-152.

[13] A. B. Kuijlaars and M. Vanlessen, Universality for eigenvalue correlations from the modified Jacobi unitary ensemble, Int. Math. Res. Not. 30 (2002), 1575-1600.

[14] N. S. Landkof, Foundations of Modern Potential Theory, Springer-Verlag, Berlin-New York, 1972.

[15] Y. Last and B. Simon, Fine structure of the zeros of orthogonal polynomials, IV. A priori bounds and clock behavior, to appear in Comm. Pure Appl. Math.

[16] E. Levin and D. S. Lubinsky, Applications of universality limits to zeros and reproducing kernels of orthogonal polynomials, to appear in J. Approx. Theory.

[17] D. S. Lubinksy, A new approach to universality limits involving orthogonal polynomials, to appear in Ann. of Math.

[18] D. S. Lubinsky, A new approach to universality limits at the edge of the spectrum, preprint.

[19] A. Máté and P. Nevai, Bernstein's inequality in $L^{p}$ for $0<p<1$ and $(C, 1)$ bounds for orthogonal polynomials, Ann. of Math. (2) 111 (1980), 145-154.

[20] A. Máté, P. Nevai, and V. Totik, Szegö's extremum problem on the unit circle, Ann. of Math. (2) 134 (1991), 433-453.

[21] P. Nevai, Orthogonal polynomials, Mem. Amer. Math. Soc. 18 (1979), no. 213, 185 pp.

[22] F. Peherstorfer, Deformation of minimal polynomials and approximation of several intervals by an inverse polynomial mapping, J. Approx. Theory $\mathbf{1 1 1}$ (2001), 180-195.

[23] F. Peherstorfer and P. Yuditskii, Asymptotic behavior of polynomials orthonormal on a homogeneous set, J. Anal. Math. 89 (2003), 113-154.

[24] E. B. Saff and V. Totik, Logarithmic Potentials With External Eields, Grundlehren der Mathematischen Wissenschaften [Fundamental Principles of Mathematical Sciences], 316, Springer-Verlag, Berlin, 1997.

[25] B. Simon, Orthogonal Polynomials on the Unit Circle, Part 1: Classical Theory, AMS Colloquium Series, American Mathematical Society, Providence, RI, 2005.

[26] B. Simon, Orthogonal Polynomials on the Unit Circle, Part 2: Spectral Theory, AMS Colloquium Series, American Mathematical Society, Providence, RI, 2005.

[27] B. Simon, Fine structure of the zeros of orthogonal polynomials, I. A tale of two pictures, Electron. Trans. Numer. Anal. 25 (2006), 328-268.

[28] B. Simon, Fine structure of the zeros of orthogonal polynomials, III. Periodic recursion coefficients, Comm. Pure Appl. Math. 59 (2005) 1042-1062.

[29] B. Simon, Rank one perturbations and the zeros of paraorthogonal polynomials on the unit circle, to appear in J. Math. Anal. Appl. 
[30] B. Simon, Equilibrium measures and capacities in spectral theory, in preparation

[31] B. Simon, Szegö's Theorem and Its Descendants: Spectral Theory for $L^{2}$ Perturbations of Orthogonal Polynomials, in preparation; to be published by Princeton University Press.

[32] B. Simon, Weak convergence of CD kernels and applications, in preparation.

[33] M. Sodin and P. Yuditskii, Almost periodic Jacobi matrices with homogeneous spectrum, infinite-dimensional Jacobi inversion, and Hardy spaces of character-automorphic functions, J. Geom. Anal. 7 (1997), 387-435.

[34] H. Stahl and V. Totik, General Orthogonal Polynomials, Encyclopedia of Mathematics and its Applications, 43, Cambridge University Press, Cambridge, 1992.

[35] G. Szegö, Bemerkungen zu einer Arbeit von Herrn M. Fekete: Über die Verteilung der Wurzeln bei gewissen algebraischen Gleichungen mit ganzzahligen Koeffizienten, Math. Z. 21 (1924), 203-208.

[36] G. Szegő, Orthogonal Polynomials, Amer. Math. Soc. Colloq. Publ., Vol. 23, American Mathematical Society, Providence, R.I., 1939; 3rd edition, 1967.

[37] V. Totik, Asymptotics for Christoffel functions for general measures on the real line, J. Anal. Math. 81 (2000), 283-303.

[38] V. Totik, Polynomial inverse images and polynomial inequalities, Acta Math. 187 (2001), 139-160.

[39] V. Totik, Universality and fine zero spacing on general sets, in preparation.

[40] M. Tsuji, Potential Theory in Modern Function Theory, reprinting of the 1959 original, Chelsea, New York, 1975.

[41] W. Van Assche, Invariant zero behaviour for orthogonal polynomials on compact sets of the real line, Bull. Soc. Math. Belg. Ser. B 38 (1986), 1-13.

[42] J. L. Walsh, Interpolation and Approximation by Rational Functions in the Complex Domain, fourth ed., American Mathematical Society Colloquium Publications, Vol. XX, American Mathematical Society, Providence, R.I., 1965.

[43] H. Widom, Polynomials associated with measures in the complex plane, J. Math. Mech. 16 (1967), 997-1013.

[44] M.-W. L. Wong, First and second kind paraorthogonal polynomials and their zeros, to appear in J. Approx. Theory. 